News \& Views

\title{
Critical role of immunogenic cell death in cancer therapy
}

\author{
Tian Xia \\ Division of NanoMedicine, Department of Medicine, University of California Los Angeles, Los Angeles, CA 90095, USA \\ Center for Environmental Implications of Nanotechnology (CEIN), California NanoSystems Institute (CNSI), University of California, Los Angeles, CA 90095, USA \\ State Key Laboratory of Environmental Chemistry and Ecotoxicology, Research Center for Eco-Environmental Sciences, Chinese Academy of Sciences, Beijing 100085, China
}

In traditional cancer therapy, including chemo and radiation therapy, the goal is always to kill as much cancer cells as possible. However, the truth is that it is difficult to kill $100 \%$ of the cancer cells for these therapies and the remaining cells often leads to relapse. So the million-dollar question is, how to eliminate the majority of the remaining cancer cells so that the disease can be manageable in a clinical setting. Currently the best approach that is attracting much attention is cancer immune therapy and exciting developments in this field have led to cure in some cases that is unimaginable before [1]. There are two major parts to cancer immune therapy that need to work together to make the therapy successful. One is on the reversal of immune suppressive microenvironment in cancer to allow the immune cells to target the tumor. In cancer, there are many regulatory $\mathrm{T}$ cells (T-regs) present that secrete IL-4 and IL-10 immune suppressive cytokines, CD80 on dendritic cells bind to cytotoxic T lymphocyte-associated protein 4 (CTLA4) to inhibit T cell functions, and the tumor cells often express programed cell death ligand 1 (PD-L1) to prevent the PD1 expressing $T$ cells from attacking them. The other part is on triggering of immune responses by tumor. Tumor often fails to trigger strong activation of the immune system to kill the cancer cells, due to low antigenicity and/or adjuvanticity. For the latter part, there are rapid developments focusing on the effective triggers of immune responses, especially through a process named immunogenic cell death (ICD) [2].

What exactly is immunogenic cell death? We all know that cell death can be categorized into mainly two types, apoptosis and necrosis. The general consensus is that apoptosis is a form of programmed cell death that is non-immunogenic or tolerogenic, while necrosis is uncontrolled cell death that is highly immunogenic $[1,2]$. However, recent developments in cell death research have shown this simple categorization is largely wrong, and immunogenicity is not dependent upon a specific type of cell death. This is clearly demonstrated in cancer cells undergoing accidental necrosis in response to freeze-thawing or boiling, although supposedly to be immunogenic, these cells are unable to activate dendritic cells and fail to elicit protective immune responses upon inoculation in syngeneic mice. Against conventional wisdom, anthracyclins (e.g., doxorubicin) triggers apoptosis in tumor cells

E-mail address: txia@ucla.edu through caspase 3 activation, yet these dying cells could elicit an effective antitumor immune response that suppresses the growth of inoculated tumors or leads to the regression of established neoplasia. Caspase inhibition by Z-VAD-fmk did not inhibit doxorubicin-induced cell death, yet suppressed the immunogenicity of dying tumor cells in several tumor animal models [3]. This brings about the concept of immunogenic cell death (ICD) in cancer, regardless of the types of cell death.

Mounting a successful immune response requires mainly two key factors, antigenicity and adjuvanticity. The first one is the presentation of neo-antigen expressed on tumor cell surface that differentiates from the self antigens. For cancer cells that harbor many oncogene mutations, there are many novel antigens that are expressed on tumor cells but not expressed on normal cells, which is especially true for advance stage of cancers. However, the presence of neo-antigen is not enough to trigger strong immune responses. The other factor that plays a major role in ICD is the production of damage-associated molecular patterns (DAMPs), which interact with pattern recognition receptors (PRRs) on monocytes, macrophages, and dendritic cells (DCs) that generates optimal conditions for initiation of antigen-specific immune responses (Fig. 1). DAMPs are different from the exogenous microorganism-associated molecular pattern (MAMPs) such as lipopolysaccharide (LPS), they are endogenous entities that serve as adjuvant to enhance immune responses to neo-antigens including the ones from cancer cells. Cancer drugs including doxorubicin, mitoxantrone, oxaliplatin and bortezomid, could trigger cellular stress that generate signals to trigger immune responses, however, other drugs including cisplatin, camptothecin, etoposide, mitomycin C, etc. failed to do so. The ICD inducing drugs could induce cellular stress including endoplasmic reticulum (ER) stress and induction of autophagy, a mechanism to restore the homeostasis in cells. Drug induced ER stress will trigger the unfolded protein response (UPR) to alleviate this stress and restore ER homeostasis, promoting cell survival. However, ER stress also leads to the relocation of ER chaperones such as calreticulin and heat shock proteins 70 and $90 \mathrm{kDa}$ to plasma membrane of the dying cancer cells [4]. These proteins serve as "eat-me" signals, promoting uptake of the cancer cell corpses and debris by DCs. Autophagy, as a general mechanism of maintaining cell homeostasis and preventing cell death, is also induced for cells under chemotherapy, 

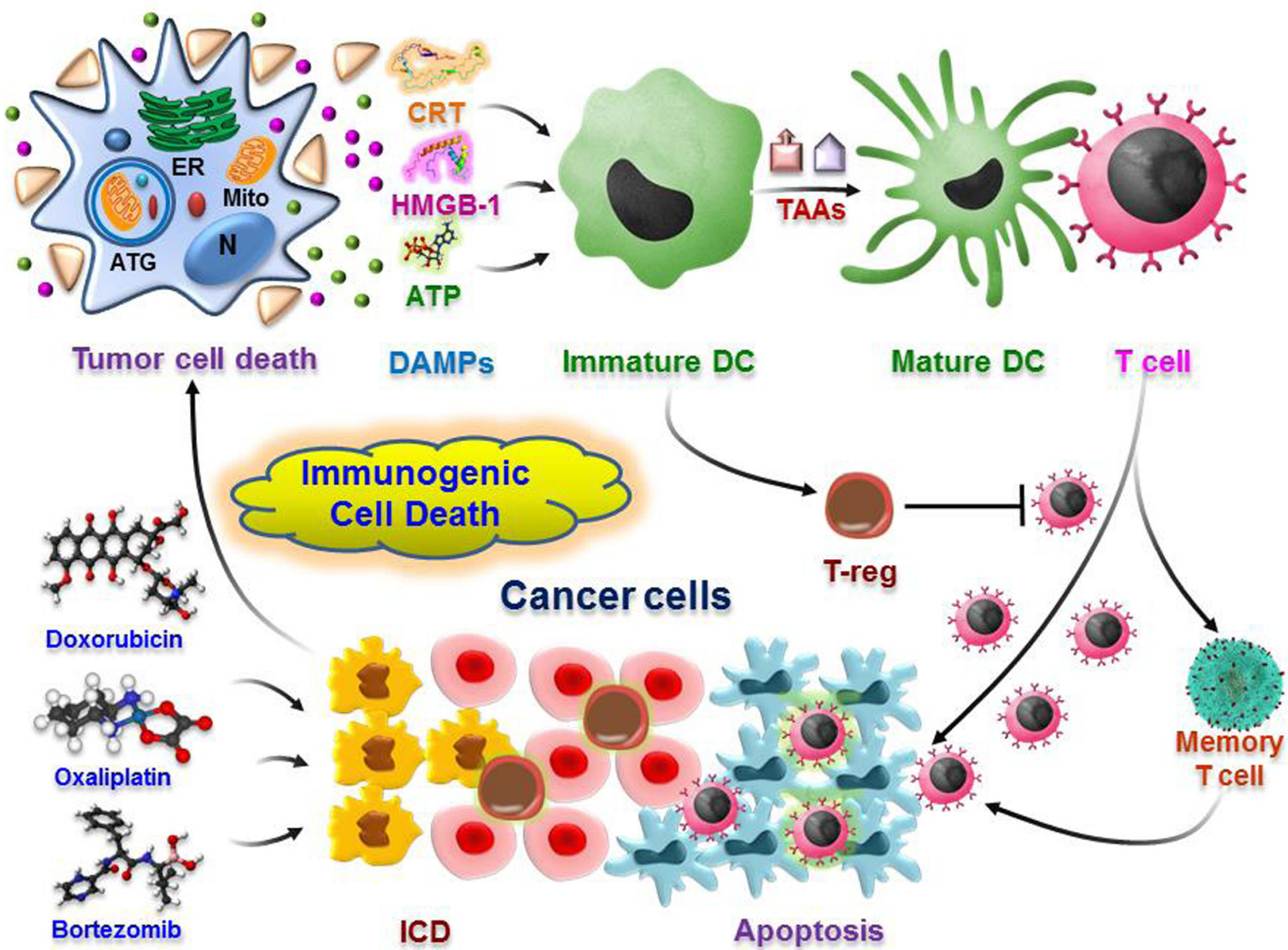

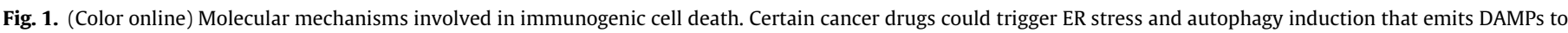

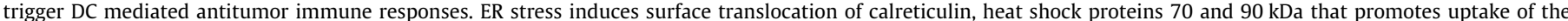

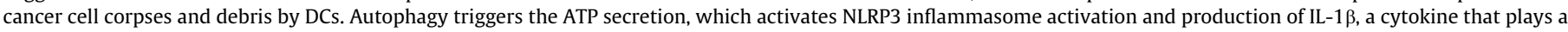

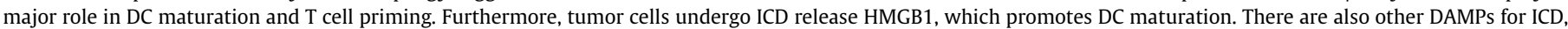

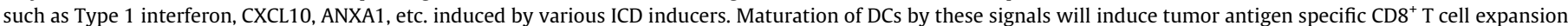
that home into the tumor site to induce cancer cell apoptosis.

which triggers the ATP secretion, and ATP serves as an adjuvant that interacts with purinergic receptor P2X7 on DCs to induce $\mathrm{K}^{+}$ efflux, leading to NLRP3 inflammasome activation and IL-1 $\beta$ production, a cytokine that plays a major role in dendritic cell maturation and antigen-specific T cell priming [5]. Furthermore, tumor cells undergo ICD release high mobility group box 1 (HMGB1), which is an important chromatin protein that interacts with nucleosomes, transcription factors, and histones, however, it gets released during apoptosis to promote DC maturation. In addition to these DAMPs, there are also other signals for ICD, such as Type 1 interferon, CXCL10, ANXA1, etc., which are shown to play important roles under various ICD triggers including hypericin-based photodynamic therapy, high hydrostatic pressure, or necroptosis. In recent years, it has become clear that many therapeutics that had been used for decades in the clinic are efficient because they engage the host immune system against malignant cells [6]. ICD is one of the several mechanisms by which chemotherapy, targeted anticancer agents and certain radiation therapy can elicit tumor specific immune responses [6].

Substantial efforts have been devoted to reinvigorate the immune responses in cancer patients. The most successful approach is to block the immune suppressive receptors in activated $\mathrm{T}$ cells, such as CTLA4 and PD- 1 by monoclonal antibodies (mAbs). Three checkpoint blockers, the CTLA4-targeting mAb ipilimumab and the PD-1-targeting mAbs nivolumab and pembrolizumab, were approved by the US Food and Drug Administration and its counterparts in other countries. Despite the success, there are many cases that the checkpoint blockers alone could not achieve the desired therapeutic outcomes, especially to the cancers that have low antigenicity and/or adjuvanticity. However, recent clinical trials showed that combining these checkpoint blockers with chemotherapeutic agent could improve the clinical outcomes of various cancers. Attempts are being made to induce immunogenicity in otherwise non-immunogenic cancers by inducing ICD, which had a positive impact on overall survival in cohorts of breast, colorectal, head and neck, and hepatocellular carcinoma patients [7]. All these data indicate that ICD is an integral part of anticancer immune therapy.

Despite the progress in our understanding of ICD and its critical role in cancer therapy, cancer cells, during the evolution of the disease, develops multiple strategies to evade immunosurveillance. This includes inhibition of various processes involved in antigen presentation, DAMP emission and sensing. These strategies include tumor heterogeneity may change the antigen profiles, low mutational load of the cancer cells, enhanced ER homeostasis, overexpression of BCL-2 proteins, defective cellular stress responses due to loss of functions (HMGB1 loss, P53 mutations, P2X7 mutation in ATP signaling, autophagy gene BECN downregulation, low 
calreticulin levels, etc.). Furthermore, our understanding of relationships between cancer therapeutics and ICD is still patchy. There is an urgent need to have a systemic survey of currently available anticancer agents to elicit the ICD in human cancer cells of different origins. Furthermore, it is important to identify novel agents that could elicit ICD or convert the non-immunogenic cell death into a bona fide ICD [8]. In addition, together with ICD, combination therapy including checkpoint blockers has the high probability to achieve more efficient therapeutic effects in clinical settings [6]. In this effort, nanoparticle-based immunotherapeutic strategies for the treatment of cancer, serving as antigen/adjuvant delivery vehicles and their combinations with chemotherapeutics, could further facilitate the development of the field [8-10].

\section{References}

[1] Galluzzi L, Buque A, Kepp O, et al. Immunogenic cell death in cancer and infectious disease. Nat Rev Immunol 2017:17:97-111.
[2] Kroemer G, Galluzzi L, Kepp O, et al. Immunogenic cell death in cancer therapy. Annu Rev Immunol 2013;31:51-72.

[3] Casares N, Pequignot MO, Tesniere A, et al. Caspase-dependent immunogenicity of doxorubicin-induced tumor cell death. J Exp Med 2005;202:1691-701.

[4] Obeid M, Tesniere A, Ghiringhelli F, et al. Calreticulin exposure dictates the immunogenicity of cancer cell death. Nat Med 2007;13:54-61.

[5] Guo Z, Zhang M, An H, et al. Fas ligation induces IL-1beta-dependent maturation and IL-1beta-independent survival of dendritic cells: different roles of ERK and NF-kappaB signaling pathways. Blood 2003;102:4441-7.

[6] Bezu L, Gomes-de-Silva LC, Dewitte H, et al. Combinatorial strategies for the induction of immunogenic cell death. Front Immunol 2015;6:187.

[7] Menger L, Vacchelli E, Adjemian S, et al. Cardiac glycosides exert anticancer effects by inducing immunogenic cell death. Sci Transl Med 2012;4. 143ra199.

8] Son KJ, Choi KR, Ryu CK, et al. Induction of immunogenic cell death of tumors by newly synthesized heterocyclic quinone derivative. PLoS ONE 2017;12: e0173121.

[9] Zhao X, Yang K, Zhao R, et al. Inducing enhanced immunogenic cell death with nanocarrier-based drug delivery systems for pancreatic cancer therapy. Biomaterials 2016;102:187-97.

[10] Shao K, Singha S, Clemente-Casares X, et al. Nanoparticle-based immunotherapy for cancer. ACS Nano 2015:9:16-30. 